\title{
Fernerkundung und GIS bei Umweltmonitoring und Umweltmanagement
}

\begin{abstract}
Remote sensing image analysis systems and geographic information systems (GIS) show great promise for the integration of a wide variety of spatial information as a support to environmental monitoring and management tasks. Current and future remote sensing programs are based on a variety of sensors that will provide timely and repetitive multisensor earth observation data on a global scale. GIS offer efficient tools for handling, manipulating, analyzing and presenting spatial data that are required for environmentally sound decision making. To combine the power of both spatial technologies, however, efficient synergistic processing techniques have to be developed to cope with large multisensor image datasets and to automatically extract information for environmental GIS applications. These efforts have to be put in a broader perspective of an evolving discipline Geoinformatics as one component of a new and innovative interdisciplinary approach for academic education. One example of this approach is the new academic program Environmental Sciences at the University of Vechta.
\end{abstract}

\section{Einleitung}

1987 veröffentlichte die World Commission on Environment and Development ihren Bericht «Our Common Future». Ein zentrales Element dieses Berichtes war die Vorstellung und Unterstützung des Konzeptes zur nachhaltigen Entwicklung (sustainable development) als Ziel der zukünftig von den Vereinten Nationen zu verfolgenden Entwicklungspolitik. Nachhaltige Entwicklung wurde definiert als «Entwicklung, die die Ansprüche der heutigen Generation befriedigt, ohne die Chancen zukünftiger Generationen zu schmälern, ihre eigenen Entwicklungsziele erreichen zu können». Um ein solches Planungs- und Managementskonzept ausführen zu können, welches die mittel- und langfristigen Effekte auf unsere Umwelt berücksichtigt, ist es zunächst notwendig, genügend Informationen über den jetzigen Zustand unserer Umwelt zu besitzen, um Planer und Entscheidungsträger in den Zustand zu versetzen, intelligente und dem Anspruch der nachhaltigen Entwicklung genügende Entscheidungen treffen zu können.

Tatsächlich sind in weiten Teilen der Welt nur ungenügende, veraltete, ungenaue oder auch gar keine Informationen über den jeweiligen Zustand der Umwelt vorhanden. Ein weiteres Problem ist das der Datenverfügbarkeit und des Datenzugriffs. In der United Nations Conference on Environment and Development (UNCED) in Rio de Janeiro im Jahre 1992 wurde die Agenda 21 - An Action Plan for the 21st Century aufgestellt. Im Kapitel 40, Information for Decision Making, wurde der Mangel an verfügbaren, aktuellen und adäquaten Umweltinformationen beklagt und die dadurch verursachten fundamentalen Probleme, umweltverträgliche und einer nachhaltigen Entwicklung dienende Planungsentscheidungen treffen $\mathrm{zu}$ können (ESTES et al. 1992).

\section{Daten, Informationen, Entscheidungen}

Viele der Daten, die wir benötigen, um umweltbewußte Entscheidungen treffen zu können, sind entweder vorhanden oder werden von aufzeichnenden Systemen permanent gewonnen. Es muß allerdings festgehalten werden, daß Datensammeln allein noch keine Informationsfindung darstellt. Auch trifft es keineswegs zu, daß sich aus exakter und aktueller Information die «richtige» Entscheidung quasi automatisch zwingend ergibt. Von dem Meer an Daten, dem wir täglich gegenüberstehen, bleiben nur noch Flïsse von Informationen und Pfützen des Wissens übrig, wenn es zur Entscheidungsebene kommt.

Während die Fernerkundung in ihrer ursprünglichen Ausrichtung eine immerfort sprudelnde Datenquelle darstellt, benötigen wir zur Aufbereitung Systeme, die aus aufgenommenen Rohdaten Informationen erstellen. Bei raumbezogenen Daten sind es Geographische Informationssysteme oder Geo-Informationssysteme (GIS), die diese Aufgabe übernehmen können. Zum effektiven Durchführen von Managementaufgaben wäre als dritter Bestandteil dieser technologischen Komponente ein System zur Entscheidungsunterstützung (spatial decision support system - SDSS) zu nennen (Abb. 1). Ein SDSS hätte die Aufgabe, Information mit Entscheidungsmodellen bzw. Expertenwissen zu koppeln, um so Entscheidungsträgern Alternativen und Szenarien für umweltgerechte Planungen vor Augen führen zu können (CZERANKA 1995, JANKOWSKI 1995).

Manfred Ehlers, Prof. Dr., ISPA, Universität Vechta, D-49364 Vechta, mehlers@ispa.uni-vechta.de 


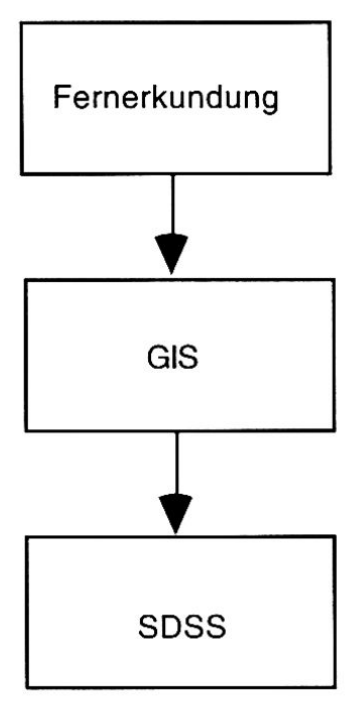

Datenaufnahme -verarbeitung

Datenspeicherung -manipulation -präsentation

Planung Entscheidung

Abb.1 Fernerkundung, GIS und SDSS in Entscheidungsprozessen.

Während SDSS zurzeit noch eher einen Forschungsgegenstand darstellen, so sind Fernerkundung und GIS heutzutage operationelle Techniken, die die Grundlagen bilden können, um eine notwendige Informationsbasis für Entscheidungsträger zu liefern. Die Fernerkundung ist eine operationelle und kontinuierliche Datenquelle für synoptische, aktuelle und quantifizierbare Information über den Zustand der Erdoberfläche; Geographische Informationssysteme liefern die notwendige Komponente zur Speicherung, Analyse und Präsentation von umweltrelevanten Informationen.

\section{Fernerkundung zum Umweltmonitoring}

Fernerkundung wird im allgemeinen definiert als «die Ermittlung von Informationen über entfernte Objekte, ohne mit ihnen in Berührung zu kommen». Dabei werden zur Informationsübermittlung im allgemeinen elektromagnetische Kräftefelder und zur Informationsaufzeichnung bilderzeugende Sensoren verwendet. Wellenlängen elektromagnetischer Kräftefelder, die zur Informationsübertragung genutzt werden, liegen zumeist im sichtbaren und nahen infraroten Bereich (Filmkameras, elektrooptische Abtaster), im thermalen Infrarot (Thermalabtaster) oder im Mikrowellenbereich (Radarsysteme). Die Aufnahmen der Fernerkundungssensoren, insbesondere bei den satellitengestützten Systemen, sind damit in der Lage, multisensorale, multitemporale und multispektrale Abbildungen des Zustandes unserer Erde zu gewinnen und der weiteren Verarbeitung zugänglich zu machen.

Eine Vielzahl von satellitengestützen Fernerkundungssystemen sind gegenwärtig operationell verfügbar. Die meistgenutzten Systeme stellen ohne Zweifel die elektrooptischen Abtaster vom Typ Landsat und SPOT dar (EHLERS 1995). Dies sind passive Systeme, die im sichtbaren und infraroten Bereich des elektromagnetischen Spektrums arbeiten. Reflektierte Solarenergie bzw. emittierte Thermalenergie wird von bildaufzeichnenden Sensoren aufgenommen und quantifiziert (SABINS 1986). Darüber hinaus werden in zunehmendem Maße aktive Sensoren eingesetzt, die im Mikrowellenbereich arbeiten (Radarsysteme). Hauptanwendungsgebiete von Radarsatellitensystemen wie ERS und JERS sind Geologie, Hydrologie oder Meereskunde. Tabelle 1 gibt einen Überblick über den jetzigen Stand der Satellitensysteme mit hoher (räumlicher) Bodenauflösung.

Weitere Satellitenmissionen beinhalten die Nachfolger der jetzigen Programme (Landsat, SPOT, ERS, IRS) und neue Sensoren wie das US Earth Observing System (EOS) mit einer Vielzahl von geplanten Sensoren, deren Spezifikationen sich allerdings beinahe jährlich ändern. Auffällig ist, daß Nationen wie Indien, Brasilien oder China nationale Satellitenprogramme gestartet haben, die sie von den nordamerikanischen oder europäischen Daten unabhängig machen. Andere interessante Entwicklungen sind Erdbeobachtungssatelliten aus der ehemaligen Spionagetechnologie mit extrem hoher räumlicher Auflösung (1-5 m) und die Bemühungen Rußlands, Bilddaten der ehemaligen sowjetischen Spionagesatelliten mit einer Auflösung von etwa $2 \mathrm{~m}$ zu vermarkten (MAREK und WEIGELT 1993, HENDERSON 1995).

Für die vorhandenen und zu erwartenden multiplen Fernerkundungsdatensätze müssen allerdings adäquate Speicherungs-, Zugriffs- und Verarbeitungsmethoden entwickelt werden, um das Potential dieser Technologie effektiv für Umweltmonitoring und -management nutzen zu können. Ohne intelligente raumbezogene Datenbank-, Analyse- und Modellierungssoftware wären nur neue Datenfriedhöfe im Terabyte-Bereich zu erwarten.

\section{GIS im Umweltmanagement}

Ein entscheidendes Ziel beim Einsatz von Informationssystemen ist die Umwandlung von Daten in Informationen. Messungen und Beobachtungen sollen in Wissen umgewandelt werden, welches die Auswahl von Optionen bei Entscheidungsprozessen unterstützt. Daten können dabei als Input in einen informationserzeugenden Prozeß gedeutet werden. Tägliche Niederschlagsmessungen können z. B. direkt als Quelle für die Berechnung von mittleren Monatswerten für einen gegebenen Ort dienen. Sie können auch als Input für ein Niederschlagsmodell genutzt werden, wobei das Ergebnis dieses Modells als Information für einen Entscheidungsproze $B$ im Umweltmanagement dienen kann (Abb. 2).

Bei Anwendungen im Umweltbereich und bei der Entwicklung nachhaltiger Planungsmethoden ist notwendigerweise die Kopplung von raumbezogener Information (z. B. Koordinaten) und attributärer Information (z. B. 


\begin{tabular}{|c|c|c|c|c|c|}
\hline $\begin{array}{c}\text { Plattform / } \\
\text { Jahr }\end{array}$ & Sensor & $\begin{array}{c}\text { Spektrale } \\
\text { Kanäle }\end{array}$ & $\begin{array}{c}\text { Spektral- } \\
\text { bereich }\end{array}$ & $\begin{array}{l}\text { Boden- } \\
\text { auflösung }\end{array}$ & Land \\
\hline $\begin{array}{c}\text { Landsat } \\
4 / 5 \\
82 / 84\end{array}$ & $\begin{array}{c}\text { MSS } \\
\text { TM }\end{array}$ & $\begin{array}{l}4 \\
7\end{array}$ & $\begin{array}{l}\text { VIS/NIR } \\
\text { VIS/NIR/ } \\
\text { MIR/TIR }\end{array}$ & $\begin{array}{c}80 \mathrm{~m} \\
30 \mathrm{~m} \\
\text { TIR: } 120 \mathrm{~m}\end{array}$ & USA \\
\hline $\begin{array}{c}\text { SPOT } \\
1 / 2 / 3 \\
86 / 90 / 93\end{array}$ & $\begin{array}{c}\text { HRV-P } \\
\text { HRV-XS }\end{array}$ & $\begin{array}{l}1 \\
3\end{array}$ & $\begin{array}{c}\text { PAN } \\
\text { VIS / NIR }\end{array}$ & $\begin{array}{l}10 \mathrm{~m} \\
20 \mathrm{~m}\end{array}$ & Frankreich \\
\hline $\begin{array}{l}\text { MOS- } \\
1 \mathrm{a} / 1 \mathrm{~b} \\
87 / 91\end{array}$ & MESSR & 4 & VIS/NIR & $50 \mathrm{~m}$ & Japan \\
\hline $\begin{array}{c}\text { IRS- } \\
1 \mathrm{~A} / 1 \mathrm{~B} / 1 \mathrm{C} \\
88 / 91 / 96\end{array}$ & LISS & $\begin{array}{l}4 \\
1 \\
4\end{array}$ & $\begin{array}{c}\text { VIS/NIR } \\
\text { PAN/ } \\
\text { VIS / NIR }\end{array}$ & $\begin{array}{l}36,5 \\
5,8 \mathrm{~m} \\
20 \mathrm{~m}\end{array}$ & Indien \\
\hline $\begin{array}{c}\text { ERS-1/-2 } \\
91 / 95\end{array}$ & SAR & 1 & MW & $30 \mathrm{~m}$ & ESA \\
\hline $\begin{array}{c}\text { JERS-1 } \\
92\end{array}$ & SAR & $\begin{array}{l}1 \\
8\end{array}$ & $\begin{array}{c}\text { MW } \\
\text { VIS/NIR }\end{array}$ & $\begin{array}{c}25 \mathrm{~m} \\
18 \times 24 \mathrm{~m}\end{array}$ & Japan \\
\hline $\begin{array}{c}\text { Shuttle / } \\
\text { Priroda } \\
93 / 94 / 96\end{array}$ & $\begin{array}{l}\text { MOMS } \\
\text { SIR-C } \\
\text { X-SAR }\end{array}$ & $\begin{array}{c}3 / 4 \\
1 \\
2 \\
1\end{array}$ & $\begin{array}{c}\text { VIS/NIR } \\
\text { PAN } \\
\text { MW } \\
\text { MW }\end{array}$ & $\begin{array}{c}12,8 / 25 \mathrm{~m} \\
4,2 \mathrm{~m} \\
20 \mathrm{~m} \\
20 \mathrm{~m}\end{array}$ & $\begin{array}{c}\text { Deutschland } \\
\text { USA } \\
\text { Italien / } \\
\text { Deutschland }\end{array}$ \\
\hline $\begin{array}{c}\text { Radarsat } \\
95\end{array}$ & SAR & 1 & MW & $15 \mathrm{~m}$ & Kanada \\
\hline
\end{tabular}

Tabelle 1 Hochauflösende Satellitenmissionen (Stand: 1996). Die Abkürzungen im Spektralbereich bedeuten: VIS = sichtbar, $\mathrm{NIR}=$ nahes Infrarot, $\mathrm{MIR}=$ mittleres Infrarot, $\mathrm{TIR}=$ thermales Infrarot, $\mathrm{MW}=$ Mikrowellenbereich, $\mathrm{PAN}=$ panchromatisch (s/w).

ein bestimmter Meßwert an einer Meßstation) zu berücksichtigen. Diese Kopplung von spatialer und nichtspatialer Information ist aber gerade das Kennzeichen von Geographischen Informationssystemen. Unterstützt von der explosionsartigen Entwicklung im Computerbereich, der Verbesserung der Benutzerschnittstellen und den Fortschritten in der Datenbankentwicklung, liefert die GIS-Technologie das ideale Werkzeug zum Handling der komplexen Datengrundlagen, um ein zielgerichtetes und effizientes Management von umweltbezogenen Informationen zu ermöglichen (EHLERS 1996).

Oftmals werden allerdings die Analysewerkzeuge eines GIS in diesem Prozeß gar nicht oder nur rudimentär genutzt. Bei vielen Anwendungen drängt sich die Beobachtung auf, daß ein GIS nur zur Datenhaltung und Visualisierung benutzt wird. Die analytischen Stärken eines GIS werden dabei wegen zu großer Komplexität vernachlässigt. Als «maßgebliche» analytische Funktionen kom- men oftmals nur simple Pufferungen und Verschneidungen zum Einsatz. Modellierungsoperationen wie bei Tomlins Map-Algebra sind dazu noch in einer recht kryptischen Sprache beschrieben, die etliche mathematische und logische Vorkenntnisse voraussetzt (TOMLIN 1990). Es ist zu hoffen, daß verstärkte Forschung im Gebiet der Taxonomie von universellen GIS-Operationen und zur Verbesserung der GIS-Benutzerschnittstellen dem Anwender ein leichteres Umgehen mit dem Werkzeug GIS ermöglichen wird. Vorschläge dazu finden sich z. B. bei ALBRECHT und EHLERS (1994).

Im Status der Forschung finden sich auch die integrierte Einbeziehung von Umweltmodellen in GIS (DEURSEN 1995, GOODCHILD et al. 1993, GOODCHILD et al. 1996) oder die Erweiterung eines GIS zum raumbezogenen Entscheidungsunterstützungssystem (CZERANKA 1996, DENSHAM et al. 1995). 
Measurement

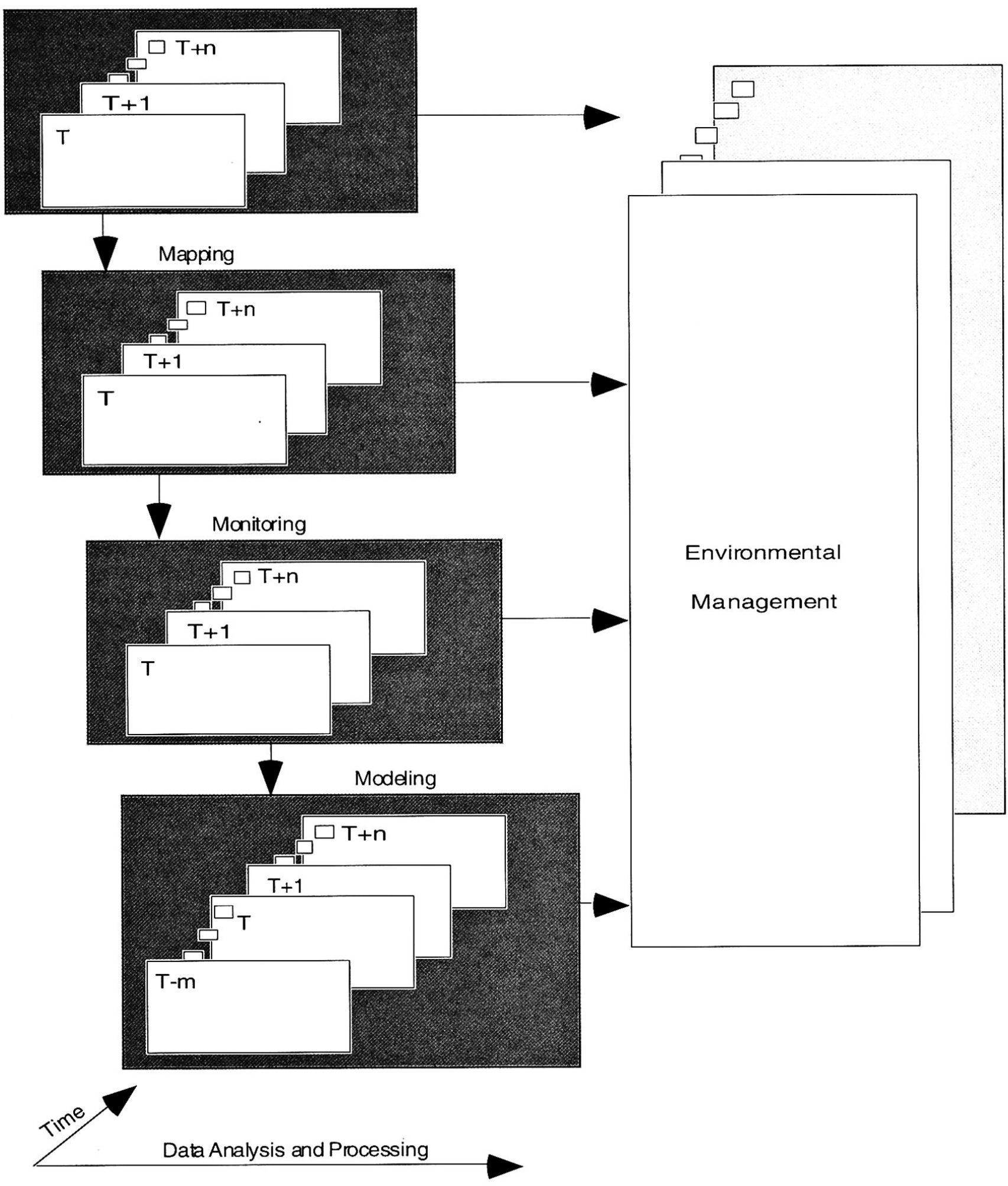

Abb.2 Umwandlung von Daten in Informationen beim Umweltmanagement: Messung (measurement), Kartierung (mapping), Monitoring und Modellierung (modeling). 


\section{Integration von Fernerkundung und GIS}

GIS und Fernerkundung (mit der dazugehörigen digitalen Bildverarbeitung) sind zwei Techniken, die sich mit der Erfassung, Speicherung, Verarbeitung und Präsentation von Geo-Information befassen. Es wäre daher nur natürlich, sie als eine Einheit zu sehen. Trotz der intensiven Diskussion um die Integration dieser Technologien existieren integrierte Systeme (IGIS) bisher erst in Ansätzen. Dies hat zum einen seinen Ursprung im historischen Hintergrund der beiden Disziplinen (Kartographie $=$ Vektor; Bildverarbeitung $=$ Raster $)$. Zum anderen sind bei raumbezogenen Phänomenen die Modellierungsansprüche verschieden: während die Behandlung von Häusern, Grundstücken usw. einen objektbasierten, im Vektorbereich besser zu modellierenden Ansatz ( $o b$ ject-view) nahelegt, sind viele natürliche Prozesse eher durch einen Feldansatz (field-view) im Rasterbereich zu realisieren (EHLERS et al. 1989). Zwar sind durchaus Fortschritte bei der Raster-Vektor-Konvertierung zu verzeichnen, eine kombinierte Prozessierungsstrategie ohne Konvertierung (zumeist in den Rasterbereich) ist allerdings auf dem Markt der integrierten GIS/Fernerkundungssysteme noch nicht zu sehen.

Konzeptionelle Probleme bei der Integration dieser beiden Disziplinen liegen vor allem in vier Bereichen: 1. Strukturen, 2. Semantik, 3. Fehlermodellierung und 4. Implementierung (EHLERS 1996). Diese vier Gebiete sind allerdings ebenfalls die Schwerpunkte der Forschung der "reinen» GIS- bzw. Fernerkundungs-/Bildverarbeitungsdisziplinen (Abb. 3). So gibt es eine große Anzahl von Forschern, die an den Themen am oberen oder unteren Rand des «Forschungswürfels» in Abb. 3 arbeiten. Nur eine kleine Anzahl von Wissenschaftern mit Forschungsinteressen, die sowohl GIS wie auch Fernerkundung umfassen, widmet sich dem zentralen Teil des Würfels.

Ansätze zu einer stärkeren Integration in Richtung einer neuen Disziplin Geoinformatik, Geomatik oder Geographische Informationswissenschaft finden sich z. B. bei EHLERS (1993), GOODCHILD (1992) und GROOT (1989).

\section{Geoinformatik in Umweltstudiengängen}

Es ist entscheidend für die Akzeptanz von Geoinformatikkomponenten für Umweltmonitoring und Umweltmanagement, daß diese Technologien in vorhandene und/ oder neue umweltbezogene Studiengänge eingebunden werden. Dieses ist am sinnvollsten in einem interdisziplinären Ansatz zu verwirklichen, der allerdings über einen "akademischen Flickenteppich" hinausgehen muß. Die deutsche Hochschullandschaft ist bisher stark durch monodisziplinäre Ansätze geprägt und beginnt eher zögerlich, Beispielen aus dem angelsächsischen Raum zu folgen, in dem Interdisziplinarität schon seit Jahrzehnten üblich ist. Die dort vorhandenen erfolgreichen Studiengänge in Environmental Sciences sind beispielhaft für manche der jetzt erfolgenden Anstrengungen im deutschsprachigen Raum.

Hier scheinen es interessanterweise eher die kleinen und mittleren Hochschulen zu sein, die innovative Wege beschreiten. Aus der Not eines begrenzten Lehrangebotes versuchen sie eine Tugend zu machen, indem sie von Beginn an integrierte Studiengänge aufbauen. In Niedersachsen sind es die drei Universitäten Oldenburg, Lüneburg und Vechta, die Diplomstudiengänge in Umweltwissenschaften anbieten. Allerdings stellt die Geoinformatik einzig in Vechta einen Schwerpunkt in der Ausbildung dar (CZERANKA und EHLERS 1994). Weitere Komponenten der Vechtaer Umweltwissenschaften sind Landschaftsökologie, Regionalwissenschaften, Raumund Umweltplanung sowie Grundlagen aus den Bereichen Jura und Management. Gleichzeitig wurde bei der Planung des Studienganges sichergestellt, daß alle Komponenten gezielt für den Inhalt des Studiums (Umweltwissenschaften) geplant und dementsprechend mit neuen Lehrinhalten (und neuem Lehrpersonal) entwickelt wurden. Erst die Zukunft wird allerdings zeigen, wie integrierte Studiengänge dieser Art sich gegen die etablierten monodisziplinären werden durchsetzen können.

\section{Literatur}

ALBRECHT, J., EHLERS, M. (1994): Virtual GIS. In: Nievergelt, J., T. Roos, H.-J. Schek and P. Widmayer (Eds.), IGIS '94: GIS, Springer Verlag, Berlin-Heidelberg, pp. 55-58.

CZERANKA, M. (1995): Is GIS Technology Reasonably Exploited for Decision Support in Ecologically-Oriented Spatial Planning? In: Kremers, H., und W. Pillmann (Eds.), Raum und Zeit in Umweltinformationssystemen, Metropolis-Verlag, Marburg, pp. 65-78.

CZERANKA, M. (1996): Spatial Decision Support Systems in Naturschutz und Landschaftspflege? GIS in Naturschutz und Landschaftspflege, Bayerische Akademie für Naturschutz und Landschaftspflege, 4/96, pp. 21-28. 
CZERANKA, M, EHLERS, M. (1994): Designing a Post Graduate Program in Environmental Monitoring: The Role of GIS. Proceedings, 5th European Conference on Geographical Information Systems (EGIS/MARI'94), Paris, France, pp. 252-261.

DENSHAM, P. J., ARMSTRONG, M. P., KEMP, K. K. (1996): Collaborative Spatial Decision-Making. Technical Report 95-14, NCGIA, $184 \mathrm{pp}$.

V. DEURSEN, W.P.A. (1995): GIS and Dynamic Models. Nederlandse Geografische Studies 190, Utrecht, 198 pp.

EHLERS, M. (1993): Integration of GIS, Remote Sensing, Photogrammetry and Cartography: The Geoinformatics Approach. Geo-Informations-Systeme, 6, No. 5, pp.18-23.

EHLERS, M. (1995): Integrating Remote Sensing and GIS for Environmental Monitoring and Modeling: Where are we? Geo Info Systems, July 1995, pp. 36-43.

EHLERS, M. (1996): Remote Sensing and GIS: Advanced Technologies for Environmental Monitoring and Management. In: Singroy, V. H., D. D. Nebert and A. I. Johnson (Eds.), Remote Sensing and GIS for Site Characterization, ASTM Publication STP1279, pp.17-25.

EHLERS, M., EDWARDS, G., BEDARD, Y. (1989): Integration of Remote Sensing with Geographic Information Systems: A Necessary Evolution. Photogrammetric Engineering and Remote Sensing, 55, No. 11, pp.1619-1627.

ESTES, J. E., EHLERS, M., MALINGREAU, J.P., NOBLE, I.R., RAPER, J., SELLMAN, A., STAR, J.L., WEBER, J. (1992): Advanced Data Acquisition and Analysis Technologies for
Sustainable Development. Man and Biosphere (MAB) Digest 12, Unesco, Paris, $68 \mathrm{pp}$.

GOODCHILD, M. F. (1992): Geographic Information Science. International Journal of GIS, 6, No. 1, pp. 31-45.

GOODCHILD, M.F., PARKS, B.O., STEYART, L.T. (Eds.) (1993): Environmental Modeling with GIS. Oxford University Press, New York-Oxford, 488 pp.

GOODCHILD, M. F., STEYART, L.T., PARKS, B. O., JOHNSTON, C., CRANE, M., GLENDENNING, S. (Eds.) (1996): GIS and Environmental Modeling: Progress and Research Issues. GIS World Books, Fort Collins, $486 \mathrm{pp}$.

GROOT, R. (1989): Educational Requirements in Geomatics. ITC Journal, pp.1-4.

HENDERSON, F.B. (1995): Remote Sensing for GIS. GIS World, 8, No. 2, pp. 42-44.

JANKOWSKI, P. (1995): Integrating GIS and Multiple Criteria Decision-Making Methods. International Journal of GIS, 9, No. 3, pp. 251-273.

MAREK, K.H., WEIGELT, A. (1993): The 3rd Generation of Space Photography. Proceedings, ISPRS WG IV/2 Workshop and Conference "International Mapping from Space", Schriftenreihe des Instituts für Photogrammetrie, Universität Hannover, 15, pp.101-106.

SABINS, F. F. (1986): Remote Sensing. Principles and Interpretation. Freeman and Company, New York, 449 pp.

TOMLIN, C. D. (1990): Geographic Information Systems and Cartographic Modelling. Prentice Hall. Englewood Cliffs, N. J., 249 pp. 